\title{
Evaluation of Generation Expansion Investment under Competitive Market Environment
}

\author{
Jifeng Su, Student Member, IEEE, and Felix F. Wu, Fellow, IEEE
}

\begin{abstract}
This paper proposes a new approach of generation expansion planning and its risk assessment under the competitive market environment. Different from the traditional probabilistic production costing (PPC) method under monopoly environment which is based on the deterministic generator loading priority list, the uncertainties of biddings for long-term contracts in electricity market are introduced into the new model. Based on the probability distribution of the surplus on bidding price over marginal cost, the distribution of expected generated energy for a given period can be achieved to calculate the profit of a generator. In order to manage the risks of the investment on generation expansion project, a risk assessment tool called Conditional Value at Risk (CVaR) is used. It gives the investors an intuitionistic criterion for making investment decision. A numerical example is given in this paper to valid the method.
\end{abstract}

Index Terms--Generation Planning, Probabilistic Production Costing, Bidding, Conditional Value at Risk (CVaR), Risk Assessment

\section{INTRODUCTION}

$\mathrm{W}$ ITH the trend of the deregulation in electricity industry, the increased uncertainty has impacted significantly on generation expansion planning of electric utilities [1] ,[2]. Different from regulated monopoly that has a guaranteed rate of return, the generating companies (GENCOs) under competitive market environment are independent entities and should plan for their own generation investment separately. The decisions are based on the profits of investment.

In the last thirty years, standard process and accepted methodologies have been developed for generation expansion for the traditional regulated power system. An engineering model that starts from a long-term load forecast, called production costing model was developed [3], [4]. By using this model, power system operation can be simulated repeatedly to obtain production costs and carry out for a period of time sufficient to cover the effect of the new generation. Equivalent Load Duration Curve (ELDC) was developed first for this approach and the Monte Carlo version of it was developed later. Forced Outage Rate (FOR) and uncertainty on load forecasting, which are considered as two

This work was supported by the grant from Research Grant Council, Hong Kong SAR.

Jifeng Su is with Centre for Electrical Energy Systems, The University of Hong Kong, Pokfulam Road, Hong Kong (e-mail: jfsu@eee.hku.hk).

Felix F. Wu is with Centre for Electrical Energy Systems, The University of Hong Kong, Pokfulam Road, Hong Kong (e-mail: ffwu@eee.hku.hk). major risks for generation expansion planning under regulated monopoly, can be taken care of well in PPC model.

The PPC model has been served as one of the important tools that are used in generation expansion planning in regulated monopoly when all generators belong to the same company. The generator loading priority list was determined by central optimized dispatch schedule under such an environment. However, for the new deregulated market environment, especially in a competitive auction market for long-term electricity contract, the bidding results will act a critical role for the determination of generator loading priority list, and influence the expected investment payoff. This paper presents a new approach on such a problem. It considers the uncertainties caused by market bidding [5]. Since the investor (GENCO) can not achieve full set of information from other competitors, an independent non-negative normal distribution was constructed as the percentage of each GENCO's surplus over its marginal cost to simulate the bidding randomness in the market. Biddings on the contracts will lead to loading priority list random. Based on a Monte Carlo simulation, the profit probability distribution of the GENCO's investment can be achieved, and a risk assessment tool called CVaR [6] can be applied to manage the risk of the generation expansion project.

To valid the method, a test system simplified from RTS96 [7] has been used and numerical results are obtained for verification.

\section{Probabilistic Production Costing Method with UNCERTAINTY OF MARKET BIDDING}

\section{A. PPC Method for Traditional Regulated Monopoly Environment}

Probabilistic production costing method was widely used in generation expansion planning under traditional regulated monopoly environment. It presents a mathematical model that used to develop the cost of operating a power system over a given time period [3]. Assume a loading priority list of the units is known. The forecasted load demand curve and the available generation capacity (or FOR) of each unit are considered to be random variables. Elementary probability theory is applied to calculate the expected generated energy.

Assume the load demand is a random variable $L$. The probability distribution function that system available capacity $x$ greater than load $L$ can be defined as

$$
F_{L}(x)=\operatorname{Pr} o b\{L \leq x\}
$$


The inverted load duration curve $G(x)$ can be interpreted as the percentage of time that load exceeds $x$, i.e.

$$
G(x)=1-F_{L}(x)
$$

For a given loading priority list of available generators in the system, after units $1,2, \ldots, k$ have been committed, the equivalent load demand $Q_{k}$ is recursively to be

$$
\left\{\begin{array}{c}
Q_{0}=L \\
Q_{k}=Q_{k-1}-A_{k} \quad k=1,2, \ldots, N
\end{array}\right.
$$

where $A_{k}$ is the available capacity of unit $k$.

Base on above definitions, the distribution function can be obtained from the convolution formula

$$
F_{Q_{k}}(x)=q_{k} F_{Q_{k-1}}(x)+p_{k} F_{Q_{k-1}}\left(x+c_{k}\right)
$$

where $q_{k}$ is the forced outage rate of unit $k, p_{k}=\left(1-q_{k}\right)$, and $c_{k}$ is the capacity of unit $k$.

If we define the equivalent inverted load duration curve after $k$ th unit have been committed as

$$
G_{k}(x)=1-F_{Q_{k}}(x)
$$

Then it can be obtained directly by the recursive formula

$$
\left\{\begin{array}{c}
G_{0}(x)=G(x) \\
G_{k}(x)=q_{k} G_{k-1}(x)+p_{k} G_{k-1}\left(x+c_{k}\right)
\end{array}\right.
$$

And the expected energy generated by unit $k$ during period $T$ will be

$$
E_{k}=p_{k} T \int_{0}^{c_{k}} G_{k-1}(x) d x
$$

Thus for a deterministic loading priority list for units, considering unit FOR and forecasted load demand curve, the expected generated energy for each unit can be obtained.

\section{B. PPC with Uncertainties of Market Bidding}

From subsection II-A, we can achieve the expected energy generated by unit $k$ during period $T$. It can be derived under deterministic loading priority list. The load forecast randomness and unit FOR have already been taken into account. But for deregulated power industry, the uncertainty of biddings among different GENCOs must be taken into account in the model. These bidding behaviors will increase the risks for generation asset investment.

Since the electricity transactions are mainly long-term contracts between GENCOs and the system operator, and the bidding price of these contracts will decide the priority of loading order, the uncertainties on these biddings shall be constructed for the PPC calculation.

To simplify the description, all the units belong to the same GENCO are combined and treated as one unit. For a given GENCO $k$, the uncertainty on bidding price of long-term electricity contracts can be assumed as a function that includes a random variable which follows a non-negative normal distribution. The percentage that bidding price over its marginal cost is the expected surplus, which can be defined as the distribution below:

$$
\text { Surplus }_{k} \sim \operatorname{abs}\left(N\left(\mu_{k}, \sigma_{k}\right)\right)
$$

where $\mu_{k}$ is the mean of bidding price surplus above marginal cost for GENCO $k$, and $\sigma_{k}$ is the volatility of the surplus for GENCO $k$. We select a non-negative distribution here because each rational GENCO will not bid prices lower than its marginal cost.

Then the bidding price of GENCO $k$ can be given as the following:

$$
P_{k}^{E}=\left(1+\text { Surplus }_{k}\right) \times \text { Cost }_{k}^{V}
$$

where $\operatorname{Cost}_{k}^{V}$ is the variable cost for generating electricity per MWh of GENCO $k$, which can be described as

$$
\operatorname{Cost}_{k}^{V}=P_{k}^{F} H_{k}+M_{k}^{V}
$$

where $P_{k}^{F}$ is the fuel price for GENCO $k, H_{k}$ is the heat rate of GENCO $k$, and $M_{k}^{V}$ is the variable maintenance cost for GENCO $k$. To simplify the problem, the fuel price in this paper is assumed to be constant for each kind of fuel, and unit heat rate is assumed to be 1 .

For a system with $N$ GENCOs including the new one which will be invested, $N$ independent bidding price stochastic process for a long-term electricity contract can be constructed. The loading priority list $L P L$, which is the priory to generate electricity, can be achieved by sorting these prices in an ascending order,

$$
L P L=\left\{G E N C O_{k} \mid k=\operatorname{Sort}_{\text {ascending }}\left(P_{k}^{E}\right)\right\}
$$

By applying the formulas in above subsection, the expected generated energy during contract period $T$ for the generator that will be invested can be easily calculated. And the uncertainties from load forecast, unit FOR and market bidding have already been taken into account in the model to meet the requirements of market environment.

\section{RISK ASSESSMENT FOR GENERATION INVESTMENT}

By introducing bidding uncertainties of different GENCOs into probabilistic production costing model, a loading priority list can be achieved, and expected generated energy $E_{k}$ can be calculated during period $T$.

Base on $E_{k}$, the revenue for GENCO $k$ during contract period $T$ can be given as the following:

$$
R_{k}=E_{k} \cdot\left(P_{k}^{E}-\text { Cost }_{k}^{V}\right)-M_{k}^{C}
$$

where $R_{k}$ is the revenue of GENCO $k, M_{k}^{C}$ is the constant maintenance cost of GENCO $k$.

Assume the investment on new generation project apportioned to period $T$ is $\operatorname{Cost}_{k}^{I}$, which will be invested on the beginning of $T$, and the finance payment for the contract will be executed at the end of period $T$. Then by applying Net Present Value (NPV) method for capital budgeting [8], the present value of profit for the generation expansion project during period $T$ shall be

$$
V_{k}=e^{-r T} R_{k}-\operatorname{Cost}_{k}^{I}
$$

where $r$ is the risk free rate of return of the market.

By using a Monte Carlo simulation approach, the probability distribution function for $E_{k}$ can be achieved.

In order to manage the risk of the investment on generation expansion project, risk assessment shall be fulfilled to help the investor making decision of whether accept or reject the project. In financial area, a risk assessment tool called Value 
at Risk (VaR) [9] and its improvement Conditional Value at Risk (CVaR) [6] have been widely used. They are suitable tools for our purpose.

Value at Risk can be defined as the follow: we are $x \%$ certain that our loss on the investment project during period $T$ in the future will not larger than $y$ dollar. This $y$ is the VaR of the investment. It can be given as the following formula:

$$
V a R_{k}=-C D F_{V_{k}}^{-1}(1-\mathrm{x} \%)
$$

where $C D F_{V_{k}}^{-1}$ is the inversed cumulative distribution function for investment profit $V_{k}$ (and the negative profit means the loss), $x \%$ is the confidence level that investor invests on the project.

As an improvement, CVaR can overcome the shortcomings of $\mathrm{VaR}$ [10]. CVaR can tell us how bad it could be, i.e. when the loss is greater than $\mathrm{VaR}$, what is the expected loss.

$$
\operatorname{CVaR}_{k}=E\left\{\operatorname{Loss}_{V_{k}} \mid \operatorname{Loss}_{V_{k}}>\operatorname{VaR}_{k}\right\}
$$

Using the probability density function and cumulative distribution function for generation investment project given by Monte Carlo simulation, these risk indexes VaR and CVaR can be calculated for long-term generation asset risk assessment.

\section{Algorithm}

As mentioned above, a succinct algorithm can be summarized in the following steps:

Step-1. Achieve data and parameters of competitive GENCOs, including the approximation of unit forced outage rate, probability distribution of expected bidding price surplus, etc. The data can be achieved by historic market experience and classic unit types.

Step-2. Get system forecasted load demand curve from Open Access Same-Time Information System (OASIS) or system operator for further calculation of probabilistic production costing.

Step-3. Sample one generator loading priority list for period $T$ according to the randomness of bidding price surplus on long-term electricity contract with respect to an approximated non-negative probability distribution.

Step-4. For the given sample, apply probabilistic production costing simulation to obtain the expected generated energy during period $T$ for invested generator.

Step-5. Calculate the revenue and profit of invested generator during period $T$ for given sample with consideration of variable and constant cost.

Step-6. Use Monte Carlo simulation to achieve the empirical probability distribution function of profit (loss) for invested generation asset during period $T$.

Step-7. Evaluate the risk of the investment on generator expansion project by applying Value at Risk and Conditional Value at Risk approach to support the decision.

\section{NumericAl EXAMPLES}

In order to valid the method, a numerical example is given in this section.
RTS 96 system is used as the data of the test system. Some simplifications are made to achieve a clearly physical meaning and to simplify the description.

The generator information of the test system is given in Table I. The units owned by the same company will be combined and treated as one large generator for simulation. Performance differences caused by unit types are ignored. These differences can be considered in a further detailed model if necessary. The variable maintenance cost and fuel cost are listed in Table II.

TABLE I

GENERATOR INFORMATION FOR TEST SYSTEM

\begin{tabular}{c|c|c|c}
\hline GENCO ID & GENCO Name & $\begin{array}{c}\text { Unit Capacity } \\
(\mathrm{MW})\end{array}$ & $\begin{array}{c}\text { Number of } \\
\text { Units }\end{array}$ \\
\hline 1 & $\mathrm{U} 50$ & 50 & 6 \\
\hline 2 & $\mathrm{U} 12$ & 12 & 5 \\
\hline 3 & $\mathrm{U} 20$ & 20 & 4 \\
\hline 4 & $\mathrm{U} 76$ & 76 & 4 \\
\hline 5 & $\mathrm{U} 100$ & 100 & 3 \\
\hline 6 & $\mathrm{U} 155$ & 155 & 4 \\
\hline 7 & $\mathrm{U} 197$ & 197 & 1 \\
\hline 8 & $\mathrm{U} 350$ & 350 & 2 \\
\hline 9 & $\mathrm{U} 400$ & 400 & \\
\hline
\end{tabular}

TABLE II

GENERATOR OPERATING PARAMETERS FOR TEST SYSTEM

\begin{tabular}{c|c|c|c|c}
\hline GENCO ID & $\begin{array}{c}\text { Forced } \\
\text { Outage } \\
\text { Rate }\end{array}$ & $\begin{array}{c}\text { Fuel cost } \\
(\$ / \mathrm{MWh})\end{array}$ & $\begin{array}{c}\mathrm{M}^{\mathrm{V}} \\
(\$ / \mathrm{MWh})\end{array}$ & $\begin{array}{c}\mathrm{M}^{\mathrm{C}} \\
\left(10^{5} \$ / \text { year }\right)\end{array}$ \\
\hline 1 & 0.01 & 0 & 0.02 & 0.3 \\
\hline 2 & 0.02 & 2.3 & 5 & 10 \\
\hline 3 & 0.1 & 3 & 5 & 0.3 \\
\hline 4 & 0.02 & 1.2 & 0.9 & 10 \\
\hline 5 & 0.04 & 2.3 & 0.8 & 8.5 \\
\hline 6 & 0.04 & 1.2 & 0.8 & 7 \\
\hline 7 & 0.05 & 2.3 & 0.7 & 5 \\
\hline 8 & 0.08 & 1.2 & 0.7 & 4.5 \\
\hline 9 & 0.12 & 0.6 & 0.3 & 5 \\
\hline
\end{tabular}

The Forecasted Load demand curve for period $T=1$ year can be shown in Fig. 1. And the daily, weekly and seasonally characteristics of demand are considered in the curve.

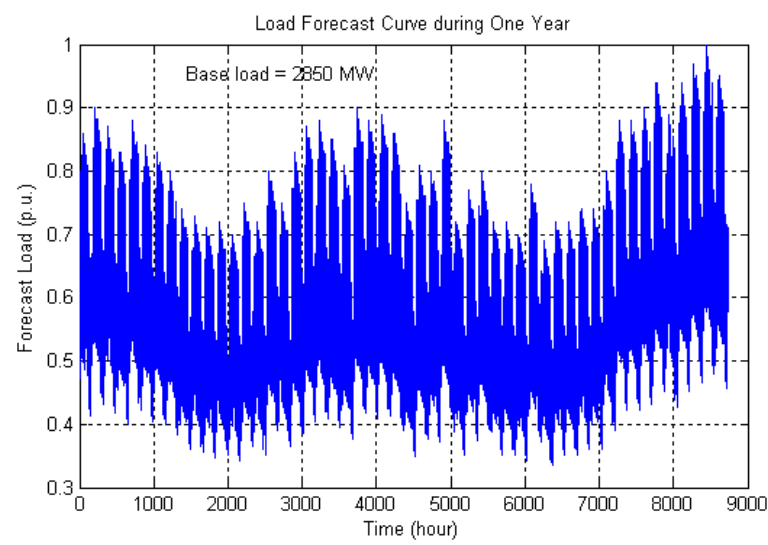

Fig. 1. Load forecast curve with daily, weekly and seasonally characteristics during period $T$. 
By applying the deterministic PPC method in the subsection II-A, for a given loading priority list for all generators in the system (includes the new one that will be invested), the expected generated energy can be achieved.

The loading priority list is generated as a sample from GENCOs bidding price surplus randomness, such as $L P L=\left\{\right.$ GENCO $\left._{k} \mid k=3,4,1,6,9,2,8,7,5\right\}$, and the unit FOR is given in Table II. Based on the forecasted load demand curve in Fig. 1, the equivalent load duration curve for each generator in the system can be calculated.

The ELDCs for GENCOs can be shown in Fig. 2. The solid lines are the ELDCs that after $k$ th GENCO have been committed to the system in order of above LPL sample from upper-right to lower-left respectively, and the dash line indicates the system ELDC.

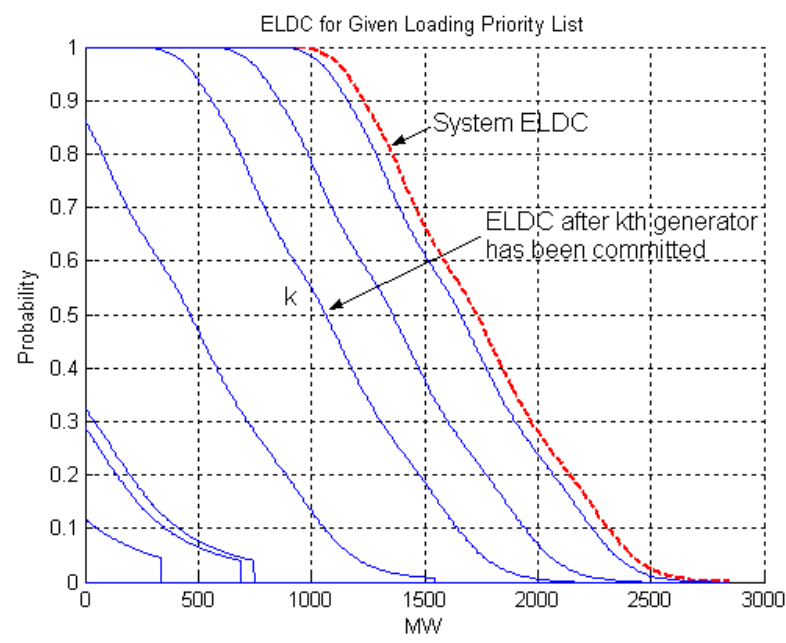

Fig. 2. Equivalent load duration curves of units for given loading priority list.

The expected generated energy for GENCOs during period $T=1$ year can be calculated and shown in the table below.

TABLE III

EXPECTED GENERATED ENERGY FOR GIVEN LOADING PRIORITY LIST $\left(\mathrm{MWH}^{*} 10^{6}\right)$

\begin{tabular}{c|c|c|c|c}
\hline GENCO & 3 & 4 & 1 & 6 \\
\hline $\mathrm{E}_{\mathrm{k}}$ & 0.6290 & 2.6026 & 2.5946 & 5.0606 \\
\hline GENCO & 9 & 2 & 8 & 7 \\
\hline $\mathrm{E}_{\mathrm{k}}$ & 3.3487 & 0.1573 & 0.5208 & 0.2094 \\
\hline
\end{tabular}

Remark: $\mathrm{E}_{5}=0$.

Assume each GENCO in the long-term contract market bids following a non-negative normal distribution as (8). In the example, suppose the surplus of bidding price follows the distribution of $a b s(N(0,0.5))$. By applying Monte Carlo simulation approach, a probability distribution of revenue for generator $k$ can be obtained. If GENCO 8 is the new generator that will be invested, the probability distribution for its revenue during period $T$ can be achieved from GENCO 8's point of view. Fig. 3 shows the empirical probability density function curve for GENCO 8 by using Monte Carlo simulation with 1000 sample set.

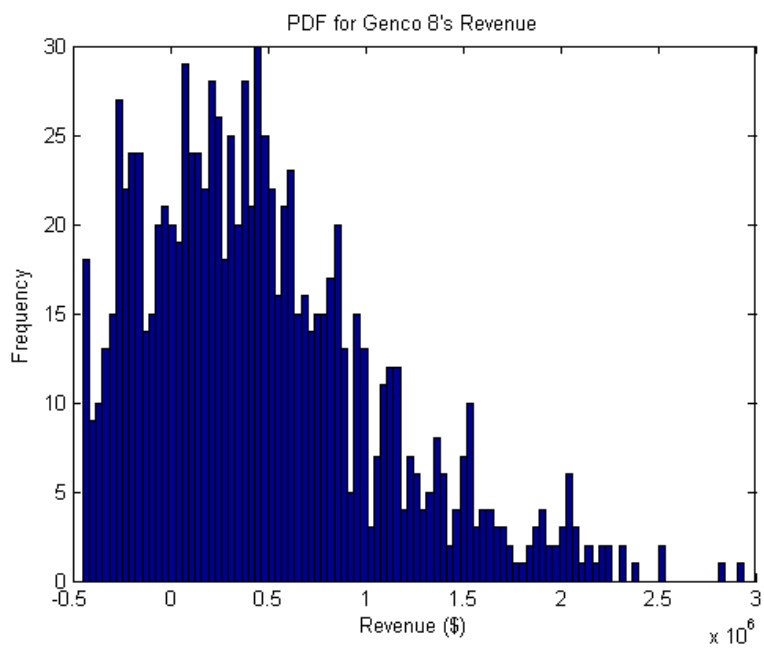

Fig 3. Empirical probability density function for GENCO 8's revenue.

If the market risk free rate of return $r=0.15$ and the apportion investment for GENCO 8 for period $T$ is $\operatorname{Cost}_{8}^{I}=$ $\$ 10^{5}$, the present value of $V_{8}$ 's profit probability distribution for GENCO 8 can be given as Fig. 4 by applying the NPV approach.

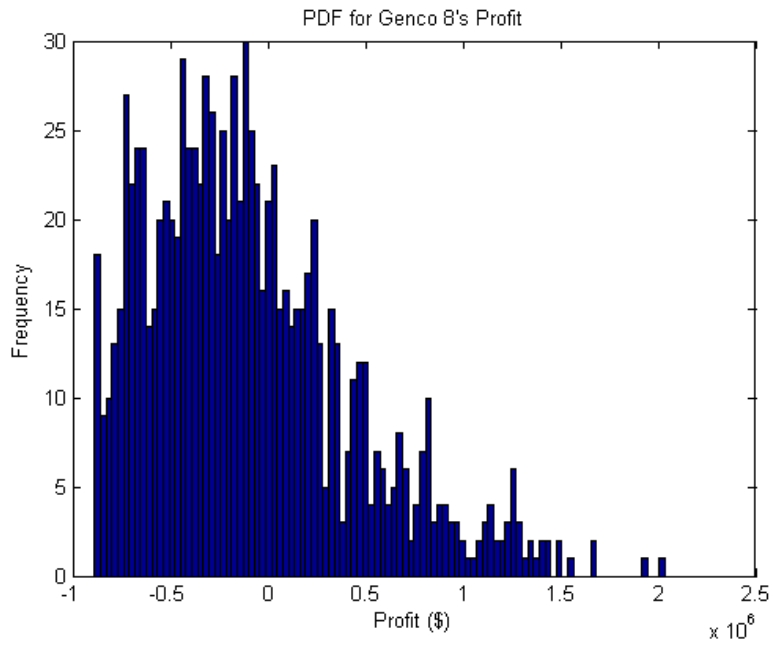

Fig. 4. Empirical probability density function for GENCO 8's profit.

By cumulating the probability density function curve in Fig. 4, we can obtain the cumulative distribution curve for GENCO 8's profit during period $T$. The cumulative distribution function (CDF) curve for $V_{8}$ is given in Fig. 5.

In order to manage the risk on the investment of GENCO 8, risk assessment tool $\mathrm{VaR}$ and $\mathrm{CVaR}$ are used to evaluate the project. Suppose that GENCO 8's payoff confidence level is $95 \%$, according to the definition in (14), the risk evaluation index $\mathrm{VaR}$ of the investment on this expansion project can be achieved on the CDF curve. The negative profit on the curve represents the loss for the investment project.

$$
\operatorname{VaR}_{8}=0.741286 \times 10^{6} \$
$$


Base on VaR, the improved index $\mathrm{CVaR}$ can be calculated as the following:

$$
C V a R=0.803617 \times 10^{6} \$,
$$

which is the expectation when loss is larger than VaR.

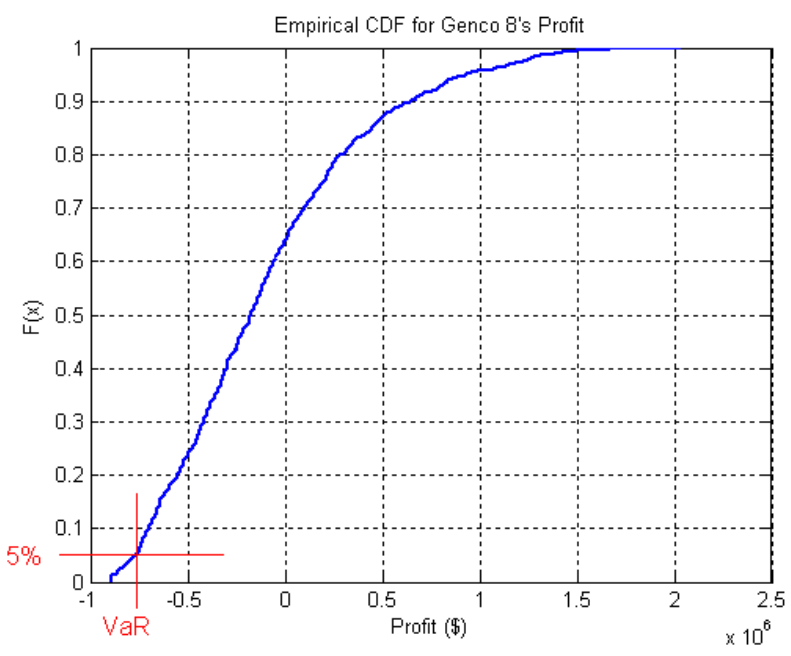

Fig. 5. Empirical cumulative distribution function for GENCO 8's profit.

With respect to different payoff confidence levels for the GENCO, a series of CVaR can be achieved and used to support the decision of generation expansion investment. Fig. 6 shows the risk assessment results.

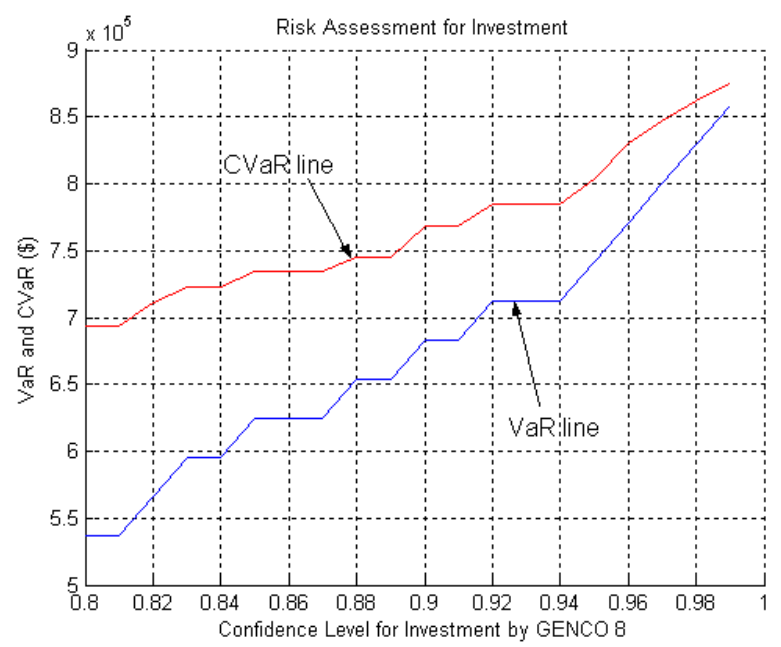

Fig. 6. Value at Risk and Conditional Value at Risk lines for different confidence level for generation expansion investment for GENCO 8.

\section{CONCLUSIONS}

This paper proposed a new approach for generation expansion planning and its risk assessment under the new deregulated environment. In order to consider the market uncertainties in generation expansion planning project for GENCOs, bidding risks for long-term contracts in the electricity market have been introduced into traditional probabilistic production costing method. Based on the probability distribution of revenue and the profit for the investment project achieved by Monte Carlo simulation, risk assessment tool conditional value at risk was applied to give the intuitionistic criterion for investment judgment. The Numerical example shows the validity and efficiency of the method.

\section{REFERENCES}

[1] M. Liu, F.F. Wu, "Generation Planning and Scheduling with Risk Management in Electricity Markets", 2003 International Conference on Electrical Engineering, Hong Kong, July 2003.

[2] A. Eydeland, K. Wolyniec, Energy and Power Risk Management: New Development in Modeling, Pricing and Hedging, Wiley, 2002.

[3] F.F. Wu, G. Gross, "Probabilistic Simulation of Power System Operation for Production Cost and Reliability Evaluation," IEEE Special Publication 77 CHII88-2 CAS, Special Sessions on Power Systems, International Symposium on Circuits and Systems, Apr. 1977, pp.877-899.

[4] A. Breiphol, F. Lee, "Comparison of Probabilistic Production Cost Simulation Methods," IEEE Transactions on Power Systems, Vol. 4, No. 4, Oct. 1989, pp.1326-1334.

[5] R. Dahlgren, C. C. Liu, J. Lawarree, "Risk Assessment in Energy Trading," IEEE Transactions on Power Systems, Vol. 18, No. 2, May 2003, pp.503-511.

[6] S. Alexander, Thomas F. Coleman, Y. Li, "Minimizing CVaR and VaR for a Portfolio of Derivatives," International Conference on Modeling, Optimization, and Risk Management in Finance, Gainesville, Mar. 5-7, 2003.

[7] Grigg, C., "The IEEE reliability test system: 1996", Paper 96 WM 326-9 PWRS, IEEE Winter Power Meeting, 1996.

[8] P. Peterson, J. Fabozzi, Capital Budgeting: Theory and Practice, New York, N.Y. Wiley, c2002.

[9] Holton, Glyn A., Value at Risk: Theory and Practice, San Diego, Calif. London Academic Press, c2003.

[10] G. Ch. Pflug, "Some Remarks on the Value-at-Risk and the Conditional Value-at-Risk", Probabilistic Constrained Optimization, Nonconvex Optim. Appl., vol. 49, Kluwer Academic Publishers, Dordrecht, 2000, pp. $272-281$

\section{BIOGRAPHIES}

Jifeng Su (SM'2004) received his MS degree from the Department of Electrical Engineering at Tianjin University, China in 2003. He is now a Ph.D candidate in the Department of Electrical and Electronic Engineering, the University of Hong Kong. His research interests are power system stability, security region visualization, electricity market, generation and transmission expansion planning and risk management $e t c$.

Felix F. Wu (F'1989) graduated from the National Taiwan University with a B.S. in 1965. In 1969 he received his M.S. from the University of Pittsburgh and his Ph.D. from the University of California, Berkeley in 1972. He is now a Chair Professor of electrical engineering at the University of Hong Kong. Prior to that he was a Professor and Vice-Chair of Department of EECS, UCB. His research interests are electric energy industry restructuring, power system investment planning, design of modern control centers, distribution automation, distributed processing etc. 